\title{
Constructing Long Short-Term Memory Networks to Predict Ulcerative Colitis Progression from Longitudinal Gut Microbiome Profiles
}

\author{
$\mathrm{Xu} \mathrm{Li}^{1}$ and Pingzhao $\mathrm{Hu}^{2}$ \\ ${ }^{1}$ Biostatistics Division, Dalla Lana School of Public Health, University of Toronto, Toronto, Canada \\ ${ }^{2}$ Bioinformatics and Statistical Genetics, University of Manitoba, Winnipeg, Canada \\ E-mail: dw.li@mail.utoronto.ca
}

Received May 15, 2020

Accepted for publication June 1, 2020

Published online September 5, 2021

\begin{abstract}
Introduction: Ulcerative colitis is an intestinal disorder with an erratic progression in which the patients suffer from capricious remissions and changeful severities. Lacking prognosis to the UC progression can lead to irrational treatments that adversely affect the patients' quality of life. Existing studies have stated a connection between gut microbiomes and UC progression. We aim to construct Long Short-Term Memory (LSTM) networks to predict UC progression (remission \& severity) from longitudinal gut microbiome data.

Methods: Using one-step and two-step modelling strategies, we develop a standard LSTM network, an encoder-decoder LSTM network, a convolutional LSTM network, and several benchmarking classifiers such as random forests. For highdimensional data, we also implement auto-encoder to select variables in addition to baseline procedures like principal component analysis. We train each model using a longitudinal microbiome data, and validate them via a 10-round set splitting approach.
\end{abstract}

Results: Each proposed model shows the potential to predict UC progression, but they do not reach an optimal level for medical utilizations. The encoder-decoder LSTM demonstrates superiority over the other classifiers while the auto-encoder outperformed the baseline variable selectors.

Conclusion: We support the capacity of LSTM networks to predict UC progression from longitudinal microbiome data, and verify the strength of autoencoder networks in selecting features from high dimensional data. 